\title{
Treatment of Vogt-Koyanagi-Harada Disease
}

\author{
Musab K. Alaql ${ }^{1}$, Akinwale A. Akinfe ${ }^{2}$, Mohammed K. AlNour ${ }^{1}$ \\ 1. Medicine, Jouf University, Sakaka, SAU 2. Opthalmology, Prince Mutaib Bin Abdulaziz Hospital, Sakaka, SAU
}

Corresponding author: Akinwale A. Akinfe, akinwaleakinfe4@gmail.com

\begin{abstract}
Vogt-Koyanagi-Harada (VKH) disease is a T-cell-mediated autoimmune inflammatory disease characterized by granulomatous panuveitis with a variety of other systemic manifestations. A 29-year-old man referred with a two-week history of pain, redness, photophobia, and blurring of vision of the right eye. The patient reported a history of tinnitus and vertigo. Ocular examination revealed that the visual acuity was hand movement oculus dextrus (OD) and 1.0 oculus sinister (OS), slight periocular depigmentation in the right eye, iris bombe in the right eye, sunset glow sign similar to Dalen-Fuchs nodules of multifocal choroiditis in the right eye, reduced fovea reflex/subtle macular edema in the right eye, and normal anterior and posterior segment OS. The patient underwent a series of investigations and treatments, including corticosteroids, cyclosporine, antibiotics, and other local eye drugs. Surgical treatment included scheduling intravitreal ranibizumab for the right eye. Outcomes included improved general health conditions and improved visual condition (visual acuity improved to $0.8 \mathrm{OD}$ ). The combined therapy of immunosuppressive drugs with steroids was effective in improving visual impairment.
\end{abstract}

Categories: Ophthalmology

Keywords: autoimmune, uveitis, systemic, granulomatous

\section{Introduction}

Vogt-Koyanagi-Harada (VKH) disease is an autoimmune inflammatory disorder that affects multiple systems with ocular, auditory, skin, and neurologic involvement [1]. It was described independently by Vogt, Koyanagi, and Harada as bilateral uveitis, exudative retinal detachments, integumentary disorders, and neurologic abnormalities [2]. Although uncommon, VKH represents 7\% to $8 \%$ of patients with uveitis in Japan [3]. It is more common in Asian, Middle Eastern, Hispanic, and Native American populations [4]. An immunogenetic predisposition that is common in certain ethnic groups is more likely to be associated with VKH. The age of onset has ranged from 3 to 89 years, as studies reported, but the highest frequency was in people in their fourth decade of life $[5,6]$.

Gender plays a role in the epidemiology of VKH. Women are twice as likely to be affected by VKH than men $[7,8]$. However, some other authors found that gender is not a risk factor for VKH $[9,10]$. We present a rare case of VKH disease illustrating that the combined therapy of immunosuppressive drugs with steroids was effective in improving the visual impairment.

Received 06/09/2020

Review began 07/06/2020 Review ended 07/06/2020 Published 07/11/2020

\section{(c) Copyright 2020}

Alaql et al. This is an open access article distributed under the terms of the Creative Commons Attribution License CC-BY 4.0., which permits unrestricted use, distribution, and reproduction in any medium, provided the original author and source are credited.

\section{Case Presentation}

A 26-year-old man was referred with a two-week history of pain, redness, photophobia, and blurring of vision of the right eye. There was associated history of tinnitus and vertigo. There was no history suggestive of meningismus; however, there was malaise, fever, headache, nausea, abdominal pain, but no stiffness of the neck or back. There was slight periocular vitiligo, but no poliosis. There was no history of joint pain, and no history of oral or genital ulceration. There was no backache and no history of cough, night sweats, or chest pain. There was no prior history of ocular injury and no previous eye surgery. The patient was neither diabetic nor hypertensive. The review of systems was not contributory.

On general and systemic examination, there was no acute distress, and the man's blood pressure was 131/68 $\mathrm{mmHg}$. His pulse rate was 78 beats per minute. An ocular examination revealed that the visual acuity was hand movement oculus dextrus (OD) and 1.0 oculus sinister (OS), with a slight periocular depigmentation in the right eye, and ciliary injection in the right eye.

Applanation tonometry revealed $14 \mathrm{mmHg}$ OD/16 mmHg OS. The examination also detected multiple moderate keratic precipitates in the right eye, moderate depth anterior chamber in the right eye, iris bombe in the right eye, posterior synechia with almost seclusio pupillae in the right eye, slight cortical opacities and clear vitreous in the right eye, sunset glow sign similar to Dalen-Fuchs nodules of multifocal choroiditis in the right eye, reduced fovea reflex/subtle macular edema in the right eye, and normal anterior and posterior segment OS.

The patient was admitted for proper evaluation and treatment with a multidisciplinary approach form of management. 
Investigations included complete blood count, erythrocyte sedimentation rate, C-reactive protein, Mantoux test, venereal disease research laboratory, serial anterior segment photography, B-scan ultrasonography OD, brain computed tomography, orbit, and sinus with contrast, lumbar puncture for cerebrospinal fluid (CSF) pleocytosis, computerized visual field, optical coherence tomography, fundus fluorescein angiography/indocyanine green, and an electroretinogram.

Treatment aimed to minimize the patient's symptoms and save his eyesight through suppression of the ciliary spasm resulting in photophobia OD, suppression of the anterior segment inflammation OD, adhesiolysis OD, and treatment of macular edema and features suggestive of choroiditis. Medical treatment included intravenous (IV) methylprednisolone $1 \mathrm{~g}$ in $250 \mathrm{ml}$ saline given over 30 minutes per day for three days and then tablet prednisolone $15 \mathrm{mg}$ three times per day for three days, $10 \mathrm{mg}$ three times per day for three days, and $5 \mathrm{mg}$ three times per day for three days; IV ceftriaxone $1 \mathrm{~g}$ every 12 hours for three days, IV metronidazole $500 \mathrm{mg}$ every eight hours for three days, IV omeprazole $40 \mathrm{mg}$ daily for three days, Gutt prednisolone forte every two hours OD for seven days, Gutt Vigamox every four hours OD for seven days, Gutt Ocugesic every six hours OD for seven days, Gutt cyclosporines $0.05 \%$ (Restasis) every eight hours OD for seven days, and Gutt atropine every 12 hours OD for seven days.

Surgical treatment included an intravitreal ranibizumab injection for the right eye after obtaining informed consent for an intravitreal anti-vascular endothelial growth factor injection for the right eye (Figure 1).
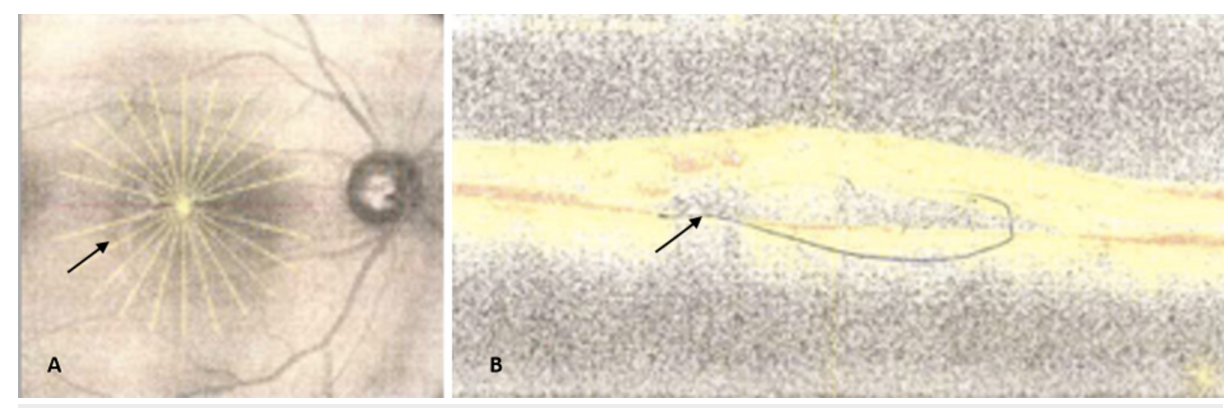

\section{FIGURE 1: (A) Right eye optical coherence tomography with obvious darkening of the fovea aspect of the macular and disc pallor. (B) Obvious early onset cystoid macula edema oculus dextrus.}

Outcomes included improved general health and improved visual conditions (visual acuity improved to 0.8 OD).

\section{Discussion}

VKH disease is characterized by bilateral uveitis as the most common initial manifestation with red eyes, blurred vision, and pain. This is usually accompanied by systemic symptoms. Auditory symptoms include vertigo, tinnitus, and deficient hearing. Neurologic symptoms include meningeal irritation and involve headache, stiffness of the neck and back, meningitis, CSF pleocytosis, cranial nerve palsies, hemiparesis, transverse myelitis, and ciliary ganglionitis. Cutaneous manifestations include poliosis, vitiligo, and alopecia. The vitiligo often is found in the sacral region [2].

The broad spectrum of symptoms found in VKH suggests that there is a central mechanism involved in the multisystemic manifestations. Currently, VKH is believed to be a T-cell-mediated autoimmune disease [11]. An antigenic component present in dermal, uveal, and meningeal melanocytes seems to be directing an autoimmune reaction. More evidence suggesting that VKH is an autoimmune disease is its association with other autoimmune disorders. These include Hashimoto thyroiditis, autoimmune polyglandular syndrome, immunoglobulin A nephropathy, and Guillain-Barré syndrome [12-15].

Clinically, VKH can be divided into four stages: prodromal, acute uveitic, convalescent, and chronic recurrent [16]. In the prodromal phase, symptoms may mimic a viral infection presented with flu-like symptoms that lasts for a few days [17]. The acute uveitic stage occurs within three to five days of the first stage as patients may experience blurred vision in both eyes due to diffuse choroiditis [17]. The convalescent stage usually follows a few months later. At this stage, depigmentation of the integument and choroid occurs [18]. The chronic stage may develop by interrupting the convalescent stage in $17 \%$ to $73 \%$ of patients $[16,17]$. Ocular complications such as cataract, choroidal neovascularization, glaucoma, and retinal fibrosis can be observed in this stage [8].

This patient's visual prognosis is generally good with prompt diagnosis and aggressive immunomodulatory treatment. Response of the inner ear to corticosteroids take one to four months and can give complete 
improvement of hearing, but corticosteroids cannot guard against effects on the eye, and it may lead to cataract, glaucoma, and optic atrophy. Skin changes typically persist despite therapy.

\section{Conclusions}

In this rare case of VKH disease, the combined therapy of immunosuppressive drugs with steroids was effective in improving our patient's visual impairment. A holistic , multidisciplinary approach to health care is warranted to optimize patient outcomes.

\section{Additional Information \\ Disclosures}

Human subjects: Consent was obtained by all participants in this study. Conflicts of interest: In compliance with the ICMJE uniform disclosure form, all authors declare the following: Payment/services info: All authors have declared that no financial support was received from any organization for the submitted work. Financial relationships: All authors have declared that they have no financial relationships at present or within the previous three years with any organizations that might have an interest in the submitted work. Other relationships: All authors have declared that there are no other relationships or activities that could appear to have influenced the submitted work.

\section{References}

1. Andreoli CM, Stephen Foster C: Vogt-Koyanagi-Harada disease. Int Ophthalmol Clin. 2006, 46:111-122. 10.1097/00004397-200604620-00011

2. Baltmr A, Lightman S, Tomkins-Netzer O: Vogt-Koyanagi-Harada syndrome: current perspectives. Clin Ophthalmol. 2016, 10:2345-2361. 10.2147/OPTH.S94866

3. Lodhi SA, Reddy JL, Peram V: Clinical spectrum and management options in Vogt-Koyanagi-Harada disease Clin Ophthalmol. 2017, 11:1399-1406. 10.2147/OPTH.S134977

4. Ohguro N, Sonoda K-H, Takeuchi M, Matsumura M, Mochizuki M: The 2009 prospective multi-center epidemiologic survey of uveitis in Japan. Jpn J Ophthalmol. 2012, 56:432-435. 10.1007/s10384-012-0158-z

5. Rathinam SR, Vijayalakshmi P, Namperumalsamy P, Nozik RA, Cunningham ET Jr: Vogt-Koyanagi-Harada syndrome in children. Ocul Immunol Inflamm. 1998, 6:155-161. 10.1076/ocii.6.3.155.4041

6. Martin TD, Rathinam SR, Cunningham ET: Prevalence, clinical characteristics, and causes of vision loss in children with Vogt-Koyanagi-Harada disease in South India. Retina. 2010, 30:1113-1121. 10.1097/IAE.0b013e3181c96a87

7. Lertsumitkul S, Whitcup SM, Nussenblatt RB, Chan C-C: Subretinal fibrosis and choroidal neovascularization in Vogt-Koyanagi-Harada syndrome. Graefes Arch Clin Exp Ophthalmol. 1999, 237:1039-1045. 10.1007/s004170050342

8. Moorthy RS, Inomata H, Rao NA: Vogt-Koyanagi-Harada syndrome. Surv Ophthalmol. 1995, 39:265-292. 10.1016/S0039-6257(05)80105-5

9. Meng Q, Liu X, Yang P, Hou S, Du L, Zhou H, Kijlstra A: PDCD1 genes may protect against extraocular manifestations in Chinese Han patients with Vogt-Koyanagi-Harada syndrome. Mol Vis. 2009, 15:386-392.

10. Hu K, Yang P, Jiang Z, Hou S, Du L, Li F: STAT4 polymorphism in a Chinese Han population with VogtKoyanagi-Harada syndrome and Behçet’s disease. Hum Immunol. 2010, 71:723-726. 10.1016/j.humimm.2010.04.007

11. Wang Y, Chan C-C: Gender differences in Vogt-Koyanagi-Harada disease and sympathetic ophthalmia. J Ophthalmol. 2014, 2014:1-8. 10.1155/2014/157803

12. Suzuki H, Isaka M, Suzuki S: Type 1 diabetes mellitus associated with Graves' disease and Vogt-KoyanagiHarada syndrome. Intern Med. 2008, 47:1241-1244. 10.2169/internalmedicine.47.0715

13. Kluger N, Mura F, Guillot B, Bessis D: Vogt-Koyanagi-Harada syndrome associated with psoriasis and autoimmune thyroid disease. Acta Derm Venereol. 2008, 88:397-398.

14. Matsuo T, Masuda I, Ota K, Yamadori I, Sunami R, Nose S: Vogt-Koyanagi-Harada syndrome in two patients with immunoglobulin A nephropathy. Acta Med Okayama. 2007, 61:305-309. 10.18926/AMO/32898

15. Najman-Vainer J, Levinson RD, Graves MC, Nguyen B-T, Engstrom RE, Holland GN: An association between Vogt-Koyanagi-Harada disease and Guillain-Barré syndrome. Am J Ophthalmol. 2001, 131:615-619. 10.1016/S0002-9394(01)00958-8

16. Cunningham ET, Rathinam SR, Tugal-Tutkun I, Muccioli C, Zierhut M: Vogt-Koyanagi-Harada disease. Ocul Immunol Inflamm. 2014, 22:249-52. 10.3109/09273948.2014.939530

17. Rao NA, Gupta A, Dustin L, et al.: Frequency of distinguishing clinical features in Vogt-Koyanagi-Harada disease. Ophthalmology. 2010, 117:591-599. 10.1016/j.ophtha.2009.08.030

18. Inomata H, Rao NA: Depigmented atrophic lesions in sunset glow fundi of Vogt-Koyanagi-Harada disease . Am J Ophthalmol. 2001, 131:607-614. 10.1016/S0002-9394(00)00851-5 